Pacific Journal of Mathematics

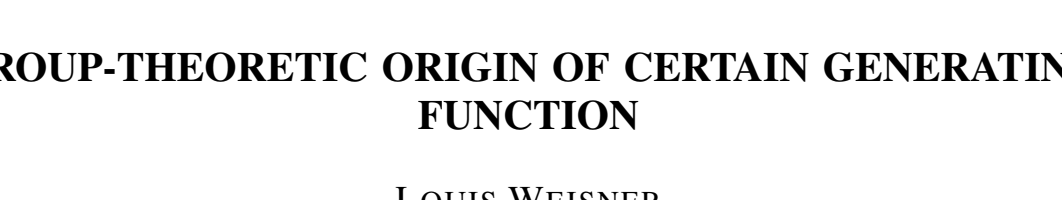




\section{GROUP-THEORETIC ORIGIN OF CERTAIN GENERATING FUNCTIONS}

LOUIS WEISNER

1. Introduction. A linear ordinary differential equation containing a parameter $n$ may be written in the form

$$
L(x, d /(d x), n) v=0 .
$$

Substituting $A=y \partial /(\partial y)$ for $n$, supposing the left member a polynomial in $n$, we construct the partial differential operator $L=L(x, d /(d x), A)$ on functions of two independent variables. This operator is independent of $n$ and is commutative with $A$. A solution of the simultaneous equation $L u=0, A u=n u$, where $n$ is a constant, has the form $u=v_{n}(x) y^{n}$, where $v=v_{n}(x)$ is a solution of (1.1). Conversely, if $v=v_{n}(x)$ is a solution of (1.1), then $u=v_{n}(x) y^{n}$ is a solution of the equations $L u=0, A u=n u$.

Now suppose that, independently of the preceding considerations, we have obtained an explicit solution $u=g(x, y)$ of $L u=0$, and that from the properties of this function we know that it has an expansion in powers of $y$ of the form

$$
g(x, y)=\sum_{n} g_{n}(x) y^{n},
$$

where $n$ is not necessarily an integer. If termwise operation with $L$ on this series is permissible, then $L$ annuls each term of the series, and $v=g_{n}(x)$ is a solution of (1.1). Thus $g(x, y)$ is a generating function for certain solutions of (1.1). The main problem is to find $g(x, y)$; its expansion is a detail of calculation.

It is difficult, in general, to find an explicit solution of $L u=0$, other than an artificial superposition of the functions $v_{n}(x) y^{n}$, for which the generating function reduces to a tautology. However, if the equation admits a group of transformations besides $x^{\prime}=x, y^{\prime}=t y(\mathrm{t} \neq 0)$, it is possible, in many cases, to find a solution which leads to a significant generating function of the form (1.2). In this paper it will be shown in detail how generating functions for the hypergeometric functions $F(-n, \beta ; \gamma ; x)$ may be obtained by this method. The Kummer functions ${ }_{1} F_{1}(-n ; \gamma ; x)$ and ${ }_{1} F_{1}(\alpha ; n+1, x)$, the Bessel functions $J_{n}(x)$ and the Hermite functions $H_{n}(x)$ admit similar treatment. The point to be emphasized is that the generating functions so obtained owe their existence to the fact that the partial differential equation derived from the ordinary differential equation in the manner described above is invariant with respect to a nontrivial continuous group of transformations.

Received February 1, 1954, and in revised form May 20, 1954. 
2. The hypergeometric functions $F(-n, \beta ; \gamma ; x)$. Suppose that $\gamma$ is not an integer; then the hypergeometric equation

$$
x(1-x) \frac{d^{2} v}{d x^{2}}+\{\gamma-(\alpha+\beta+1) x\} \frac{d v}{d x}-\alpha \beta v=0
$$

has the linearly independent solutions

$$
v_{1}=F(\alpha, \beta ; \gamma ; x) \text {, and } v_{2}=x^{1-\gamma} F(\alpha-\gamma+1, \beta-\gamma+1 ; 2-\gamma ; x) \text {. }
$$

A solution which is regular at $x=0$ is a constant multiple of $v_{1}$. Substituting $-y \partial /(\partial y)$ for $\alpha$, so that $-\alpha$ plays the ròle of the parameter $n$ of $\S 1$, we construct the operator

$$
L=x(1-x) \frac{\partial^{2}}{\partial x^{2}}+x y \frac{\partial^{2}}{\partial x \partial y}+\{\gamma-(\beta+1) x\} \frac{\partial}{\partial x}+\beta y-\frac{\partial}{\partial y} .
$$

Setting

$$
\begin{array}{r}
A=y \stackrel{\partial y}{\partial y} \quad B=y^{-1}\left(x \frac{\partial}{\partial x}-y_{-}^{\partial} \frac{\partial}{\partial y}\right), \\
C=y\left\{x(1-x) \frac{\partial}{\partial x}+y \frac{\partial}{\partial y}+\gamma-\beta x\right\},
\end{array}
$$

it may be verified that

$$
\begin{gathered}
x L=C B+A^{2}+(\gamma-1) A, \\
{[A, B]=-B, \quad[A, C]=C, \quad[C, B]=2 A+\gamma,}
\end{gathered}
$$

where $[A, B]=A B-B A$.

It follows from these relations, and may be verified by direct calculations, that $x L$ is commutative with $A, B$ and $C$ and hence with $R=$ $r_{1} A+r_{2} B+r_{3} C+r_{4}$, where the $r$ 's are arbitrary constants.

The commutator relations (2.3) show that the operators $1, A, B, C$ generate a Lie group $\Gamma$. The elements of this group may be represented in the form $e^{R}$, but are more conveniently expressed as products of a finite number of the operators $e^{a A}, e^{b B}, e^{c C}, e^{a}$, where $a$, $b, c, d$ are constants. The operator 1 generates the multiplicative group of complex numbers, while $A$ generates the group $x^{\prime}=x, y^{\prime}=t y$ $(t \neq 0)$. We shall use these two trivial groups for purposes of normalization. We find that

$$
\begin{gathered}
e^{c C} e^{b B} f(x, y)=(1-c y)^{\beta-\gamma}\{1+c(x-1) y\}^{-\beta} f(\xi, \eta), \\
\xi=\frac{x y}{\{1+c(x-1) y\}\{(1+b c) y-b\}}, \quad \eta=\frac{(1+b c) y-b}{1-c y},
\end{gathered}
$$

where $f(x, y)$ is an arbitrary function. Since $x L$ is commutative with 
$B$ and $C$, it follows that if $f(x, y)$ is annulled by $L$, so is the right member of (2.4).

3. Conjugates sets of generators of the first order. The main problem with which we shall be concerned is that of solving the simultaneous partial differential equations $L u=0, R u=0$, where $R=$ $r_{2} A+r_{2} B+r_{3} C+r_{4}$, for all choices of the ratios of the coefficients except $r_{1}=r_{2}=r_{3}=0$. A great deal of labor is saved by the following observation: If $S$ is an element of the group $\Gamma$ of $\S 2$, then $S R S^{-1}$ has the same form as $R$; and if $u$ is annulled by $L$ and $R$, then $S u$ is annulled by $L$ and $S R S^{-1}$. It is therefore sufficient for our purpose to find the functions annulled by $L$ and one operator from each of the conjugate classes into which the operators $R$ fall with respect to $\Gamma$; and to apply $\Gamma$ to these functions.

For any two linear operators $X$ and $Y$ with a common domain of operands, we have the formal expansion

$$
e^{t X} Y e^{-t X}=\sum_{k=0}^{\infty} \frac{t^{k}}{k !}[X, Y]_{k},
$$

where

$$
\begin{array}{r}
{[X, Y]_{0}=Y,[X, Y]_{1}=X Y-Y X, \text { and }[X, Y]_{k}=\left[X,[X, Y]_{k-1}\right],} \\
(k=2,3, \cdots) .
\end{array}
$$

Hence, utilizing (2.3), we have

$$
\begin{array}{ll}
e^{a A} B e^{-a A}=e^{-a} B, & e^{a A} C e^{-a A}=e^{a} C, \\
e^{b B} A e^{-b B}=A+b B, & e^{b B} C e^{-b B}=-2 b A-b^{2} B+C-b \gamma, \\
e^{c C} A e^{-c C}=A-c C, & e^{c C} B e^{-c C}=2 c A+B-c^{2} C+c \gamma .
\end{array}
$$

Despite the use of infinite series in the derivation of these operator identities, no questions of convergence arise in their application to an operand. An arbitrary function $f(x, y)$, whose partial derivatives of the first order exist, is converted by the left member of each of these identities into a function expressed in closed form with the aid of (2.4), while the right member involves only a finite number of terms.

From the preceding identities we have

$$
\left(e^{c C} e^{b B}\right) A\left(e^{c C} e^{b B}\right)^{-1}=(1+2 b c) A+b B-c(1+b c) C+b c \gamma .
$$

It follows that $R$ is a conjugate of $\lambda A+\alpha$, for suitable choices of the constants $\lambda$ and $\alpha$, except when $r_{1}^{2}+4 r_{2} r_{3}=0$. In that case it may be inferred from (3.3) and

$$
\left(e^{B} e^{-C}\right) B\left(e^{B} e^{-C}\right)^{-1}=-C
$$


that $R$ is a conjugate of $\lambda B+\alpha$. Since only the ratios of the coefficients of $R$ are essential, we shall choose $\lambda=1$.

4. Generating functions. The general solution of the simultaneous partial differential equations $L u=0,(A+\alpha) u=0$, is a linear combination, with constant coefficients, of

$$
u_{1}=y^{-\alpha} F(\alpha, \beta ; \gamma ; x), \quad u_{2}=y^{-\alpha} x^{1-\gamma} F(\alpha-\gamma+1, \beta-\gamma+1 ; 2-\gamma ; x) .
$$

It follows from (3.4) that the general solution of the equations

$$
L u=0, \quad\{(1+2 b c) A+b B-c(1+b c) C+\alpha+b c \gamma\} u=0
$$

is a linear combination, with constant coefficients, of

$$
\begin{aligned}
G_{1}(x, y)= & (1-c y)^{\alpha+\beta-\gamma}\{(1+b c) y-b\}^{-\alpha}\{1+c(x-1) y\}^{-\beta} F(\alpha, \beta ; \gamma ; \xi), \\
G_{2}(x, y)= & (x y)^{1-\gamma}(1-c y)^{\alpha+\beta-\gamma}\{(1+b c) y-b\}^{\gamma-\alpha+1}\{1+c(x-1) y\}^{\gamma-\beta+1} \\
& \cdot F(\alpha-\gamma+1, \beta-\gamma+1 ; 2-\gamma ; \xi),
\end{aligned}
$$

where $\xi$ is given by (2.4). It is sufficient to consider only $G_{1}$, as the expansion of $G_{2}$ in powers of $y$ may be obtained from that of $G_{1}$ by simple substitutions.

If $b=0$, we normalize by choosing $c=1$, so that

$$
G_{1}=y^{-\alpha}(1-y)^{\alpha+\beta-\gamma}\{1+(x-1) y\}^{-\beta} F(\alpha, \beta ; \gamma ; \underset{1+(x-1) y}{x}) .
$$

This function has an expansion of the form $\sum_{n=0}^{\infty} g_{n}(x) y^{n-x}$. As noted in $\S 1, g_{n}(x)$ must be a solution of (2.1) with $\alpha$ replaced by $\alpha-n$. Since $g_{n}(x)$ is regular at the origin it must be a constant multiple of $F(\alpha-n$, $\beta ; \gamma ; x)$. The constant is determined by setting $x=0$. Thus

$$
\begin{gathered}
(1-y)^{\alpha+\beta-\gamma}\{1+(x-1) y\}^{-\beta} F\left(\alpha, \beta ; \gamma ; \begin{array}{c}
x \\
1+(x-1) y
\end{array}\right) \\
=\sum_{n=0}^{\infty}\left(\begin{array}{c}
\gamma-\alpha+n-1 \\
n
\end{array}\right) F(\alpha-n, \beta ; \gamma ; x) y^{n},
\end{gathered}
$$

where $x \neq 1,|y|<\min \left(1,|1-x|^{-1}\right)$. The region of convergence is determined by examining the singularities of the left member.

Similarly, if $b=1$ and $c=0$, we obtain

$$
\begin{aligned}
& (1-y)^{-\alpha} F\left(\alpha, \beta ; \gamma ; \frac{-x y}{1-y}\right)=\sum_{n=0}^{\infty}\left(\begin{array}{c}
\alpha+n-1 \\
n
\end{array}\right) F(-n, \beta ; \gamma ; x) y^{n}, \\
& |y|<\min \left(1,|1-x|^{-1}\right),(\text { Feldheim [1. p. 120]). }
\end{aligned}
$$

If $b c \neq 0$, we set $b=-w^{-1}, c=1$, and obtain 


$$
\begin{gathered}
(1-y)^{\alpha+\beta-\gamma}\{1+(w-1) y\}^{-\alpha}\{1+(x-1) y\}^{-\beta} F(\alpha, \beta ; \gamma ; \zeta) \\
=\sum_{n=0}^{\infty}\left(\begin{array}{c}
\gamma+n-1 \\
n
\end{array}\right) F(-n, \alpha ; \gamma ; w) F(-n, \beta ; \gamma ; x) y^{n}, \\
w x y \quad \begin{array}{c}
(1-y)\{1-(w-1)(x-1) y\} \\
\{1+(w-1) y\}\{1+(x-1) y\}
\end{array}, \\
\zeta=\{1+(w-1) y\}\{1+(x-1) y\} \\
|y|<\min \left(1,|1-x|^{-1},|1-w|^{-1},|1-x|^{-1}|1-w|^{-1}\right) .
\end{gathered}
$$

The required coefficient in the right member is readily obtained since the left member is unaltered by the permutation $(\alpha \beta)(w x)$. The special case $w=0$ is due to Feldheim $[1$, p. 120].

In accordance with the analysis of $\S 3$, we next examine the simultaneous equations $L u=0, B u=-u$. The general solution of the latter is $u=e^{y} f(x y)$ and is annulled by $L$ if $f(X)$ satisfies the ordinary differential equation

$$
X \frac{d^{2} f}{d X^{2}}+(\gamma+X) \frac{d f}{d X}+\beta f=0
$$

Comparing with Kummer's equation

$$
x-\frac{d^{2} v}{d x^{2}}+(\gamma-x) \frac{d v}{d x}-\alpha v=0,
$$

it follows that $u$ is a linear combination, with constant coefficients, of

$$
u_{1}=e^{y} F(\beta ; \gamma ;-x y), \quad u_{2}=e^{y}(-x y)^{1-\gamma} F(\beta-\gamma+1 ; 2-\gamma ;-x y),
$$

where the customary indices in Kummer's function have been omitted. The first of these functions is regular at $x=0$, and we obtain

$$
e^{y} F(\beta ; \gamma ;-x y)=\sum_{n=0}^{\infty} \frac{1}{n !} F(-n, \beta ; \gamma ; x) y^{n}
$$

(Humbert [2, p. 64]).

Substituting $-w y$ for $y$ in the first of the functions (4.4), we obtain $e^{-w y} F(\beta ; \gamma ; w x y)$ which is annulled by $L$ and $B-w$. Operating on this function with $e^{C}$, as suggested by (3.3) with $c=1$, we obtain the left member of

$$
\begin{aligned}
(1-y)^{\beta-\gamma}\{1+(x-1) y\}^{-\beta} \exp \left\{\begin{array}{c}
-w y \\
1-y
\end{array}\right\} F(\beta ; \gamma ; & \left.\begin{array}{c}
w x y \\
(1-y)\{1+(x-1) y\}
\end{array}\right) \\
=\sum_{n=0}^{\infty} L_{n}^{(\gamma-1)}(w) F(-n, \beta ; \gamma ; x) y^{n}, & |y|<\min \left(1,|1-x|^{-1}\right) .
\end{aligned}
$$

The required coefficient in the right member is obtained by setting $x=0$ and comparing with a known generating function for the Laguerre polynomials (Szegö [4, p. 97]). 
By (3.5) and (4.4)

$$
e^{B} e^{-C}\left\{e^{y} F(\beta ; \gamma ;-x y)\right\}=\exp \left\{1-y^{-1}\right\} y^{-\gamma}(1-x)^{-\beta} F\left(\beta ; \gamma ; \frac{-x}{y(1-x)}\right)
$$

is the only linearly independent solution of $L u=0, C u=u$ that is regular at $x=0$. Its expansion, when simplified, reads

$$
e^{y}(1-x)^{-\beta} F\left(\beta ; \gamma ; \begin{array}{c}
x y \\
1-x
\end{array}\right)=\sum_{n=0}^{\infty} \frac{1}{n !} F(\gamma+n, \beta ; \gamma ; x) y^{n},
$$

We have now obtained, in normalized form, a solution of the simultaneous equations $L u=0,\left(r_{1} A+r_{2} B+r_{3} C+r_{4}\right) u=0$ for each admissible choice of the $r$ 's, and its expansion in powers of $y$. Most of the solutions are expansible in other regions of the $y$-plane than those noted; hence other generating functions may be obtained. For example, the left member of (4.2) may be written

$$
y^{-\alpha}\left(1-y^{-1}\right)^{-\alpha} F\left(\alpha, \beta ; \lambda ; \frac{x}{1-y^{-1}}\right),
$$

except for a numerical factor. This function has an expansion valid for $|y|>1$. The result, when simplified by cancelling $y^{-\alpha}$ and replacing $y$ by $y^{-1}$, reads

$$
\begin{gathered}
(1-y)^{-\alpha} F\left(\alpha, \beta ; \gamma ; \begin{array}{c}
x \\
1-y
\end{array}\right)=\sum_{n=0}^{\infty}\left(\begin{array}{c}
\alpha+n-1 \\
n
\end{array}\right) F(\alpha+n, \beta ; \gamma ; x) y^{n}, \\
|y|<\min (1,|1-x|) .
\end{gathered}
$$

5. Application to ultraspherical polynomials. Special cases of some of the preceding formulas, involving ultraspherical polynomials, may be obtained by means of the representation

$$
P_{n}^{(\lambda)}(\cos \theta)=\left(\begin{array}{c}
2 \lambda+n-1 \\
n
\end{array}\right) e^{-n i \theta} F\left(-n, \lambda ; 2 \lambda ; 1-e^{2 i \theta}\right),
$$

which may be established by comparing the differential equations satisfied by the two members of this equation. Thus we obtain from (4.3)

$$
\begin{aligned}
& \left\{1-2 y \cos (\theta-\varphi)+y^{2}\right\}^{-\lambda} F\left(\lambda, \lambda ; 2 \lambda ; \frac{-4 y \sin \theta \sin \varphi}{1-2 y \cos (\theta-\varphi)+y^{2}}\right) \\
& =\sum_{n=0}^{\infty}\left(\begin{array}{c}
2 \lambda+n-1 \\
n
\end{array}\right) P_{n}^{(\lambda)}(\cos \varphi) P_{n}^{(\lambda)}(\cos \theta) y^{n}, \quad|y|<\left|e^{i( \pm \omega \pm \theta)}\right| .
\end{aligned}
$$

Ossicini [3] expresses the left member in terms of Legendre functions of the second kind, while Watson [5] expresses the result, for $\lambda=1 / 2$, in terms of elliptic integrals. 


\section{REFERENCES}

1. E. Feldheim, Relations entre les polynomes de Jacobi, Laguerre et Hermite, Acta Math., 75 (1942), 117-138.

2. P. Humbert, Sur les polynomes de Sonine à une et deux variables, J. École Polytech., ser. 2, 24 (1924), 59-75.

3. A. Ossicini, Funzione generatrice dei prodotti di due polinomi ultrasferici, Bolletino de la Unione Matematica Italiana (3), 7 (1952), 315-320.

4. G. Szegö, Orthogonal Polynomials, Amer. Math. Soc. Colloquium Publications, 33, 1939.

5. G. N. Watson, Notes on generating functions (3): Polynomials of Legendre and Gegenbauer, J. London Math. Soc., 8 (1931), 289-292.

Hunter College of the City of New York 

.././. ./FrontMatter/paper .pdf 


\section{Pacific Journal of Mathematics}

Nesmith Cornett Ankeny and Theodore Joseph Rivlin, On a theorem of S.

Bernstei........................................ 849

Louis Auslander, The use of forms in variational calculation .......... 853

Paul Civin, Abstract Riemann sum . .......................... 861

Paul Civin, Some ergodic theorems involving two operator ............ 869

Eckford Cohen, The number of solutions of certain cubic congruence .... . 877

Richard M. Cohn, Specializations over difference field .............. 887

Jean Dieudonné, Pseudo-discriminant and Dickson invarian . . ......... 907

Ky Fan, A comparison theorem for eigenvalues of normal matrice ........ 911

Richard P. Gosselin, On the convergence behaviour of trigonometric interpolating polynomial ........................... 915

Peter K. Henrici, On generating functions of the Jacobi polynomial . . . . . . . 923

Meyer Jerison, An algebra associated with a compact grou ............ 933

Wilhelm Magnus, Infinite determinants associated with Hill's equatio . . . . . 941

G. Power and D. L. Scott-Hutton, The slow steady motion of liquid past a semi-elliptical bos................................. 953

Lyle E. Pursell, An algebraic characterization of fixed ideals in certain function ring .................................... 963

C. T. Rajagopal, Additional note on some Tauberian theorems of O. Szás . . 971 Louis Baker Rall, Error bounds for iterative solutions of Fredholm integral

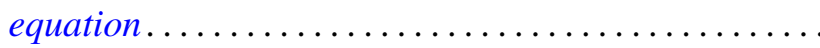

Shigeo Sasaki and Kentaro Yano, Pseudo-analytic vectors on

pseudo-Kählerian manifold ......................

Eugene Schenkman, On the tower theorem for finite group

P. Stein and John E. L. Peck, On the numerical solution of Poisson's equation over a rectangl ........................

Morgan Ward, The mappings of the positive integers into themselves which preserve divisio .

Seth Warner, Weak locally multiplicatively-convex algebra 1025

Louis Weisner, Group-theoretic origin of certain generating function .... 